\title{
Does the Canadian TIA Score adequately risk stratify patients for subsequent stroke and revascularization in the emergency department?
}

\author{
Kaitlyn Maguire ${ }^{1}$. Devin Harris ${ }^{2,3} \cdot$ David Barbic $^{3}$
}

Received: 16 July 2021 / Accepted: 29 September 2021 / Published online: 3 November 2021

() The Author(s), under exclusive licence to Canadian Association of Emergency Physicians (CAEP)/ Association Canadienne de Médecine d'Urgence (ACMU) 2021

Keywords Emergency medicine $\cdot$ Stroke $\cdot$ Transient ischemic attack $\cdot$ Canadian TIA Score

Full Citation: Perry JJ, Silvilotti ML, Édmond M, Stiell IG, Stotts G, Lee J et al. Prospective validation of Canadian TIA Score and comparison with $\mathrm{ABCD}_{2}$ and $\mathrm{ABCD}_{2}$-I for subsequent stroke risk after transient ischaemic attack: multicentre prospective cohort study. $B M J$. Feb 2021; https://doi.org/10.1136/bmj.n49

Abstract Link: https://www.bmj.com/content/372/bmj. n49

Article Type: Multicentre prospective validation study

Ratings: Methods-4.5/5, Usefulness-4/5

\section{Introduction}

\section{Background}

In patients who present with transient ischemic attack (TIA) there is an opportunity to mitigate stroke risk by identifying high-risk patients and intervening.

Kaitlyn Maguire

k.maguire@alumni.ubc.ca

1 Royal College Emergency Medicine Training Program, University of British Columbia, Vancouver, BC, Canada

2 Department of Emergency Medicine, Kelowna General Hospital, Kelowna, BC, Canada

3 Department of Emergency Medicine, University of British Columbia, Vancouver, BC, Canada

\section{Objectives}

To prospectively validate the previously derived Canadian TIA Score for stratification of stroke risk and compare the Canadian TIA Score with both $\mathrm{ABCD}_{2}$ and $\mathrm{ABCD}_{2}$-I scores.

\section{Methods}

\section{Design}

Multicentre prospective validation study.

\section{Eligibility criteria}

Adult patients with discharge diagnosis of TIA or minor stroke.

\section{Outcomes}

Primary outcome was stroke or carotid endarterectomy or carotid stenting in 7 days; secondary outcome was a stroke within 7 days, regardless of revascularization.

\section{Main results}

Over 5 years, 7,606 consecutive patients were enrolled, 108 patients experienced a subsequent stroke in 7 days, 83 had carotid endarterectomy or carotid artery stenting, 9 had both, and 34 were lost to follow-up. The Canadian TIA Score stratified the risk of stroke, revascularization or both within 7 days as low (interval likelihood ratios 0.20 , 95\% CI 0.09-0.44), medium (interval likelihood ratios 0.94, 
95\% CI 0.85-1.04) and high risk (interval likelihood ratios 2.56, 95\%CI 2.02-3.25). They found the Canadian TIA Score more accurately categorized patients into appropriate risk levels (AUC 0.70, 95\% CI 0.66-0.73) compared to the $\mathrm{ABCD}_{2}$ (AUC 0.60, 0.55-0.64), with a difference of 0.10 $(p<0.001)$ and $\mathrm{ABCD}_{2}$ - I (AUC 0.64, 95\% CI 0.59-0.68), with a difference $0.06(p=0.01)$.

\section{Appraisal}

\section{Strengths}

- Prospectively validated in both community and academic centres.

- Achieved temporal and geographic validation of previously derived score [1].

- Clinical diagnosis of TIA used to allow broader applicability.

- Large sample size used for validation by secondary outcome.

- Improved generalizability by omitting vascular imaging results in the score.

\section{Limitations}

- A composite primary outcome was used. However, the score has been validated for subsequent stroke alone, making it unlikely that this has meaningful implications.

- The score requires an online calculator.

- Further study to determine how the Canadian TIA Score will perform when applied in clinical practice is required.

\section{Context}

Patients presenting to the emergency department with TIA are at increased risk for subsequent stroke, especially within the first few days. In recent years, reductions in recurrent stroke and TIA rates have much improved with appropriate secondary stroke prevention [2]. However, identifying patients who will benefit from urgent optimization is challenging. Using a more precise risk stratification tool than was previously available may allow us to target those who will benefit from an expedited workup and specialist referral [3]. Local stroke neurologists suggest using the Canadian TIA Score. This score has been validated with excellent methodology creating a much-improved tool to predict the risk of stroke and carotid revascularization in patients presenting to the emergency department with TIA.

\section{Bottom line}

The Canadian TIA Score has outperformed other tools to risk stratify 7-day risk of stroke or carotid revascularization. This validated risk score is ready to use in the ED to inform decisions for admission, interventions and follow-up with specialists.

\section{Declarations}

Conflict of interest None

\section{References}

1. Perry JJ, Sharma M, Sivilotti ML, Sutherland J, Worster A, Émond M, et al. A prospective cohort study of patients with transient ischemic attack to identify high-risk clinical characteristics. Stroke. 2014;64(4):92-100.

2. Amarenco P, Lavallée PC, Labreuche J, Albers GW, Bornstein NM, Canhāo P, et al. One-year risk of stroke after transient ischemic attack or minor stroke. NEJM. 2016;374(16):1533-42.

3. Perry JJ, Sharma M, Sivilotti ML, Sutherland J, Symington C, Worster A, et al. Prospective validation of the $\mathrm{ABCD}_{2}$ score for patients in the emergency department with transient ischemic attack. CMAJ. 2011;183(10):1137-45. 